\title{
High temperature unfolding of Bacillus anthracis amidase-03 by molecular dynamics simulations
}

\author{
Ravi Datta Sharma ${ }^{1 *}$, Andrew M. Lynn², Pradeep Kumar Sharma1, ${ }^{1}$ Rajnee $^{3}$, Safdar Jawaid ${ }^{4}$ \\ ${ }^{1}$ Department of Microbiology, C.C.S. University, Meerut, India; ${ }^{2}$ School of Information Technology, CCBB, Jawaharlal Nehru University, \\ New Delhi, India; ${ }^{3}$ Department of Obstetrics and Gynecology, School of Medicine, West Virginia University, WV, USA; ${ }^{4}$ Department of \\ Chemistry and Biochemistry, George Mason University, Fairfax, VA, USA; \\ Ravi Datta Sharma - E-mail: ravidattasharma@gmail.com; *Corresponding author
}

Received March 21, 2009; revised April 08, 2009; accepted May 25, 2009; published July 27, 2009

\begin{abstract}
:
The stability of amidase-03 structure (a cell wall hydrolase protein) from Bacillus anthracis was studied using classical molecular dynamics (MD) simulation. This protein (GenBank accession number: NP_844822) contains an amidase-03 domain which is known to exhibit the catalytic activity of N-acetylmuramoyl-L-alanine amidase (digesting MurNAc-Lalanine linkage of bacterial cell wall). The amidase- 03 enzyme has stability at high temperature due to the core formed by the combination of several secondary structure elements made of $\beta$-sheets. We used root-mean-square-displacement (RMSD) of the simulated structure from its initial state to demonstrate the unfolding of the enzyme using its secondary structural elements. Results show that amidase- 03 unfolds in transition state ensemble (TSE). The data suggests that $\alpha$-helices unfold before $\beta$-sheets from the core during simulation.
\end{abstract}

Keywords: amidase-03; Bacillus anthracis; high temperature unfolding; hydrolase enzyme; molecular dynamics; protein unfolding

\section{Background:}

Protein folding and unfolding dynamics are still under scrutiny by the scientific community using in vivo, in vitro and in silico approaches [1-4]. Protein unfolding is one of the most fundamental biochemical processes and it is very difficult to characterize using in vivo methods. However, several theoretical methods with the use of computing power have been used in recent years to address this issue. We use molecular dynamics (MD) simulations to characterize the unfolding process of an $\mathrm{N}$ acetylmuramoyl-L-alanine amidase (PDB ID 2IR0), a cell wall hydrolase enzyme from Bacillus anthracis at high temperature. We used high temperature for this study because it is known to accelerate the unfolding path during simulation [5]. Most micro-organisms have a major cell wall component called peptidoglycan [6]. The peptidoglycan layer constantly undergoes modification by hydrolysis through the transfer of new molecules and by the release of old molecules. The hydrolysis activity is essential for the correct cell wall assembly and other metabolic activities. The enzymes performing peptidoglycan cell wall hydrolysis have been classified as $\mathrm{N}$-acetylmuramidases, N-acetylglucosaminidases and Nacetylmuramoyl-L-alanine amidases based on their substrate specificity [7].

Amidases-03 (503 residues) is a well studied enzyme protein encoded by the gene amiA present in the plasmid pXO2. It should be noted that $B$. anthracis has two virulent plasmids pXO1 and pXO2 causing cutaneous anthrax, gastrointestinal, oropharyngeal anthrax and inhalation anthrax. AmiA consists of two functional domains, of which the N-terminal one consists of three SLH (S-layer homology domain) repeats (50 residues). The remaining part of the protein is composed of two sub-domains (one domain of 100 residues with unknown structure and the other $\mathrm{N}$-acetylmuramoyl-L-alanine amidase with similarity to pfam01520 family) [7]. The amidase- 03 domain shows enzymatic activity of hydrolyzing the peptidoglycan at MurNAc-L-alanine linkage [7]. It decreases the inflammatory response and hence favoring the multiplication of B. anthracis in the host. However, some peptidoglycan hydrolases increase the inflammatory response by releasing the muramyl peptide $[\mathbf{8}, \mathbf{9}]$. The stability of yet another amidase-03 domain (GenBank accession NP 844822) is described here using data obtained from $\overline{\mathrm{MD}}$ simulations.

Methodology:

Homology model:

A homology model of amidase-03 domain (245 residues) was constructed using template PDB structure (PDB ID 1XOV) and the model is deposited in PDB with PDB ID 2IR0. The analysis shows that amidase- 03 domain is made up of $6 \alpha$-helices and $6 \beta$-sheets (see Table 1 in supplementary material). The protein contains $\beta$-sheets surrounded by the $\alpha$-helices (Figure 1a).

\section{Molecular dynamics simulation:}

Molecular dynamics simulation uses classical Newton mechanics to generate trajectories to study molecular motion using force fields. Molecular trajectories are coordinates formed by recording structural changes over time showing the positions of each atom along a series of small time step (usually $2 \mathrm{fs}$ ). The G43a1 force field with explicit SPC water and cubic boundary conditions are used to parameterize system interactions. The system consists of 8614 water molecules surrounding the protein, in a box of edge length $65.69 \AA$. This was subjected to an isobaricisothermal MD simulation at 1 atom through a Berendsen thermostat-barostat [10] with coupling constants of $0.1 \mathrm{ps}$ and $0.5 \mathrm{ps}$, respectively. The real space Coulomic and short 
range contributions were truncated at $9 \AA$ and $14 \AA$, respectively. The net negative charge on the protein was offset by replacing two water molecules with two sodium ions. This resulted in 8612 water molecules and $2 \mathrm{Na}+$ surrounding the protein. The resulting box had a density of $1.02 \mathrm{~g} / \mathrm{cc}$. All simulations were performed using a $2 \mathrm{fs}$ time step.

Energy minimization and calculation methods:

The amidase-3 model (PDB ID: 2IR0) was used as a starting structure. In an initial equilibration, the protein amidase- 03 was kept fixed, whereas the solvent and $\mathrm{Na}+$ were subjected to an energy minimization using steepest descent avoiding any steric energy conflicts. The minimum energy was achieved in 475 steps. Electrostatics is calculated using the Particle Mesh Ewald (PME) method [11]. All rigid bonds were monitored using the Lincs method [12].

\section{Simulation package and system:}

The software package used for simulation is GROMACS v 3.3.2 [13]. All simulations were performed in a desktop AMD64 4300+ processor, with 2GB RAM, using Fedora Core 8 as operating system.

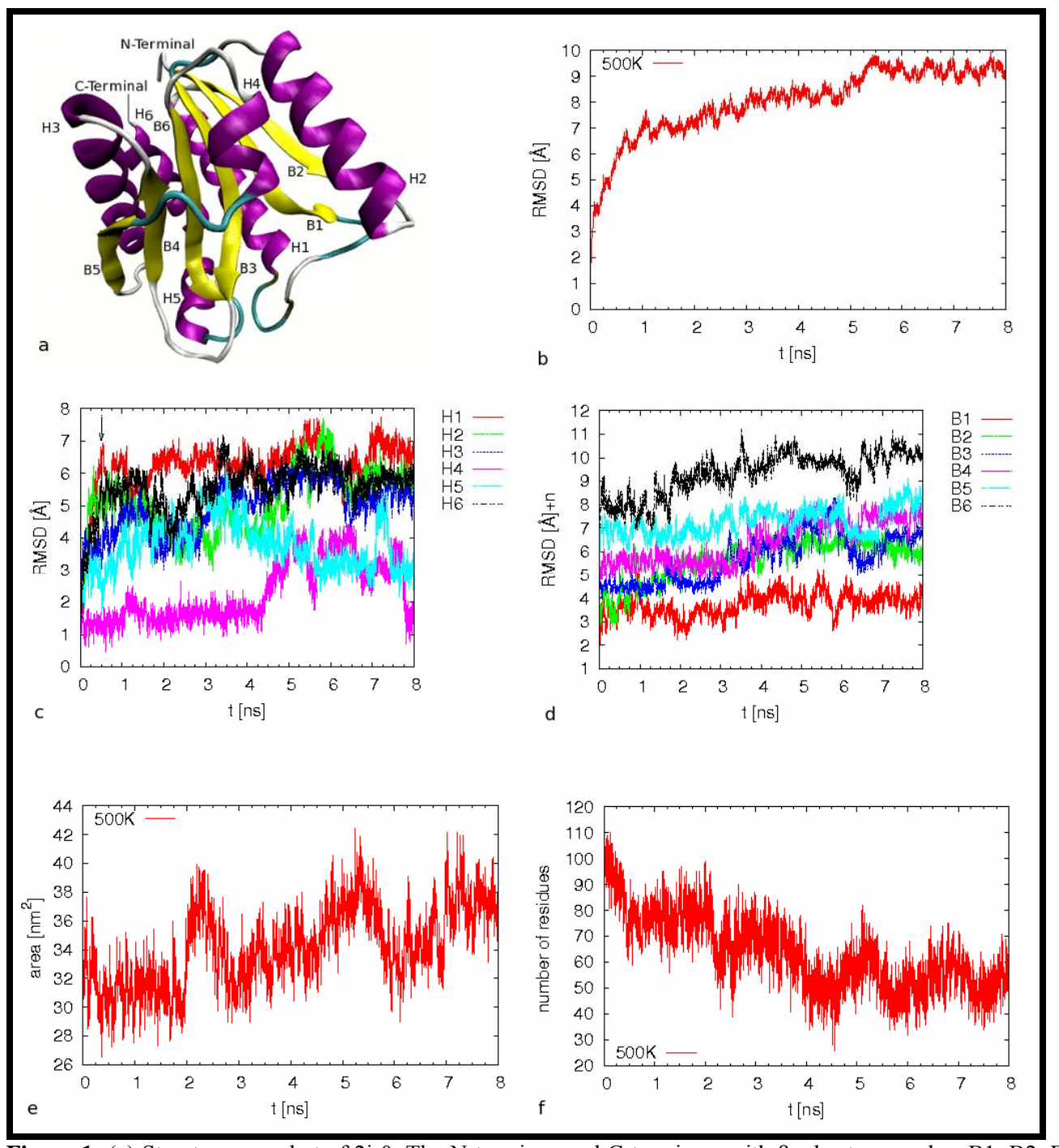

Figure 1: (a) Structure snapshot of 2ir0. The N-terminus and C-terminus, with $\beta$-sheets named as B1, B2, B3, B4, B5, B6 (forming the core inner part of the protein) surrounded by $\alpha$-helices named likewise as H1, H2, H3, H4, H5, H6; (b) Evolution of Root-mean-square-deviation $(\AA)$ of the 2 ir0 protein backbone with respect to its initial structure at $498 \mathrm{~K}$. (c) Structural changes observed in terms of RMSD in $\alpha$-helices from its native structure with time at $498 \mathrm{~K}$. (d). Structural changes observed in terms of RMSD in $\beta$-sheets from its native structure with time at $498 \mathrm{~K}$; for clarity all RMSD values are incremented by $\mathrm{n}$, where $\mathrm{n}$ is the no. of the $\beta$-sheet. (e). Variation of solvent accessible surface area correlates with time at $498 \mathrm{~K}$. (f). Decrement in total secondary structure including $\alpha$-Helix, $\beta$-Sheet, B-bridge, turn at $498 \mathrm{~K}$ confirms unfolding. 


\section{Simulation temperature:}

The protein was simulated at two temperatures $-300 \mathrm{~K}$ and $498 \mathrm{~K}$. Simulation data at high and low temperatures provide measurements for studying protein conformation stability [14]. Proteins are usually stable at $300 \mathrm{~K}$ and often undergo unfolding at $498 \mathrm{~K}$ [15].

\section{Analysis parameter:}

We used the root-mean-square-displacement (RMSD) parameter to analyze the unfolding of the protein during simulation. The RMSD of the backbone and different secondary structures of the protein were computed with respect to the initial structure of the respective trajectories after a least square fit of the non-hydrogen atoms. A cutoff value of $5 \mathrm{~ns}$ was used for equilibration simulation. The precompiled Dictionary of Protein secondary structure (DSSP) [16] was subsequently used to analyze the secondary structure unfolding, while Visual Molecular Dynamics (VMD) software [17] was used for structure visualization.

\section{Discussion:}

The amidase- 03 enzyme from B. anthracis is composed of a central core made of six $\beta$-sheets and an outer sheath of six $\alpha$-helices (Table 1). The RMSD of the protein backbone was found to be stable in the range of 2-3 $\AA$ at $300 \mathrm{~K}$, but increased to $6 \AA$ after $1 \mathrm{~ns}$ at $498 \mathrm{~K}$ (Figure $1 \mathrm{~b}$ ). The RMSD of three $\alpha$-helices (H1, H2 and H6) was similarly increased to $6 \AA$ in $0.5 \mathrm{~ns}$ time step at $498 \mathrm{~K}$. Most of the helices except $\mathrm{H} 4$ and H6 uncoil in 1 ns. The short helix H4 (residue 127 to 131) remains stable up to 4.3 ns and H6 near the inner core is stable throughout the simulation. The unfolding of the helices leads to the opening of the core to the solvent. A transition state is observed after equilibration until unfolding of $\alpha$-helices at high temperature and is described as the Transition State Ensemble (TSE) [18]. The RMSD for $\beta$-sheets increases after 2 ns until end of simulation (Figure 1d) and hence $\beta$ sheets are recorded to have greater stability than $\alpha$-helices. The collapse in the core consisting of $\beta$-sheets is accompanied by increment in backbone RMSD and is indicative of protein denaturation. This is similar to the two state reversible denaturation process described elsewhere [19]. The native structure showed RMSD $\leq 2 \AA$, the intermediate (partial unfolded) state showed $\geq 2 \AA$ RMSD $\leq 8 \AA$ (Figure 1b) during the transition state. The high RMSD value ( $\geq 8 \AA$ ) is associated with unfolded state.

Solvent accessible surface area (ASA) shows a drastic increase from 30 to $40 \mathrm{~nm}^{2}$ during $2-3 \mathrm{~ns}$ simulation (Figure 1e). This is described as the sudden unfolding of outer $\beta$-sheets (B2 and B4), and their consequent compaction. Yet another compaction found near 6 ns leads to reformation of few secondary structures. DSSP program confirms a sudden fall in total number of secondary structure including $\alpha$-helices, $\beta$-sheets, bridges and turns between 2-3 ns (Figure 1f). Nonetheless, ASA increases after 3 ns. It should be noted that the number of secondary structures with respect to initial number decreases rapidly in the first $0.5 \mathrm{~ns}$. RMSD begins to decline with unfolding of $\beta$-sheets during 2- $5.5 \mathrm{~ns}$. However, it becomes stable afterwards because the system contains small secondary structures.
The amidase- 03 enzyme unfolds in a definite hierarchy at high temperature. We observed that some secondary structures are denatured earlier than others at high temperature in the systematic analysis of trajectories at 498 $\mathrm{K}$. The most stable secondary structures are $\beta$-sheets, forming inner core of the protein surrounded by six helices. Similar path of unfolding has been reported in SH3 domain [20]. It has been reported that $\beta$-sheets denature more quickly than helices [5]. Tsai et al. (1999) [20] discussed these $\beta$-sheets are stabilized by hydrogen bonding within the sheets and the hydrophobic contacts. However, hydrophobic core becomes entirely unfolded within $4.2 \mathrm{~ns}$ in amidase-03. Nonetheless, helices are elements of the outer core and most of which are denatured rapidly within 1 ns during simulation. Proteins are usually denatured at high temperature and by using chemical denaturants. The molecular basis of temperature dependent unfolding is often studied using molecular dynamics simulation with force fields. The data described on amidase- 03 simulation is in agreement of the observation reported by Degget and Fersht [21] on folding.

\section{Conclusion}

The unfolding of amidase-03 from $B$. anthracis was studied using MD simulations at temperatures $300 \mathrm{~K}$ and $498 \mathrm{~K}$. The $\beta$-sheets and $\alpha$-helices of amidase- 03 are shown to play a role in unfolding at high temperature. Data show that $\alpha$-helices are less stable than $\beta$-sheets during simulation. The protein was found fairly stable for $8 \mathrm{~ns}$ simulation in this study. The amidase- 03 unfolding occurs in transition state ensemble (TSE).

\section{Acknowledgment}

We are thankful to Dr. S. T. Pasha, National Institute of Communicable Diseases (NICD), Delhi and Prof. Tasneem Fatma, Department of Bioscience, Jamia Millia Islamia, New Delhi for their support. The authors are thankful to Manish and Prerna for their help.

\section{References}

[1] V. Daggett et al., Curr. Opin. Struct. Biol. (1994) 4 (2): 291 [PMID:PMC45034]

[2] S. Vijayakumar et al., Biophys. J. (1993) $65: 2304$

[3] P. G. Bolhuis, Biophys. J. (2005) 88: 50 [PMID:PMC305027]

[4] A. R. Fersht et al., Cell (2002) 108: 573 [PMID:11909527]

[5] R. Day et al., J. Mol.Biol. (2002) 322: 189 [PMID:12215424]

[6] T. Fukushima et al., J. Biol. Chem. (2008) 283: 11117

[7] S. Mesnage et al., J. Bacteriol. (2002) 184: 331 [PMID:11741877]

[8] D. Gupta et al., J. Immunol. (1995) 155: 2620.

[9] E. R. Milohanic et al., Mol. Microbiol (2001). 39: 1212

[10] H. J. C. Berendsen et al., J. Phys. Chem. (1984) 81: 3684

[11] U. Essman et al., J. Chem. Phys. (1995) 103: 8577 [PMID:555740]

[12] B. Hess et al., J. Comp. Chem. (1997)18: 1463

[13] H. J. C. Berendsen et al., Comp. Phys. Comm. (1995) 91: 43

[14] D. O. V. Alonso et al., J. Mol. Biol. (1995) 247: 


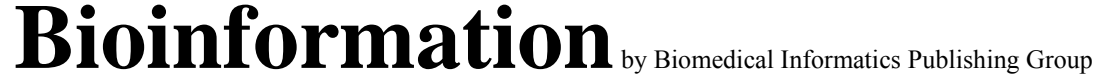

501

[15] G. Settanni et al., Biophys. J. (2008) $94: 4444$ [PMID:2480669]

[16] W. Kabsch et al., Biopolymers (1983) 22: 2577

[17] W. Humphrey et al., J. Mol. Graph., (1996) 14: 33 [PMID: 8744570]

[18] D. D. Jong et al., J. Mol. Biol. (2002) 319: 229
[19] L. S. Itzhaki et al., Proc. Natl. Acad. Sci. USA, (1995) 254: 260 [PMID: PMC40532]

[20] J. Tsai et al., J. Mol. Biol. (1999) 291: 215 [PMID: 10438616]

[21] A. R. Fersht et al., Cell (2002) 108:1 [PMID: 11909527]

Edited by $P$. Kangueane

Citation: Sharma et al, Bioinformation 3(10): 430-434 (2009)

License statement: This is an open-access article, which permits unrestricted use, distribution, and reproduction in any medium, for non-commercial purposes, provided the original author and source are credited. 


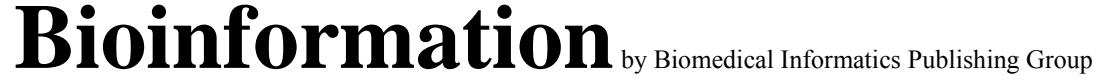

\section{Supplementary material}

Table 1: Structures designation

\begin{tabular}{|c|c|c|c|c|c|}
\hline \multirow[t]{2}{*}{ S. No. } & \multicolumn{3}{|c|}{ Residues } & \multirow[t]{2}{*}{ Structure } & \multirow[t]{2}{*}{ Name } \\
\hline & & & End & & \\
\hline 1. & 4 & 12 & & $\beta$-sheet & B1 \\
\hline 2. & 24 & 42 & & $\alpha$-helix & $\mathrm{H} 1$ \\
\hline 3. & 45 & 51 & & $\beta$-sheet & B2 \\
\hline 4. & 57 & 71 & & $\alpha$-helix & $\mathrm{H} 2$ \\
\hline 5. & 75 & 82 & & $\beta$-sheet & B3 \\
\hline 6. & 89 & 93 & & $\beta$-sheet & B4 \\
\hline 7. & 96 & 113 & & $\alpha$-helix & H3 \\
\hline 8. & 117 & 122 & & $\beta$-sheet & B5 \\
\hline 9. & 127 & 131 & & $\alpha$-helix & $\mathrm{H} 4$ \\
\hline 10. & 136 & 143 & & $\beta$-sheet & B6 \\
\hline 11. & 147 & 154 & & $\alpha$-helix & $\mathrm{H} 5$ \\
\hline 12. & 158 & 170 & & $\alpha$-helix & H6 \\
\hline
\end{tabular}

